\title{
CRENÇAS SOBRE PRÁtICAS PARENTAIS EM CRIANÇAS EM FAMÍLIAS DE CRIANÇAS COM ATRASO NO DESENVOLVIMENTO
}

\author{
Wesley Correa \\ wesleycorrea.psicologo@gmail.com \\ Maria de Fatima Minetto \\ Rafaeli Cappellaro-Kobren \\ Leandro Kruszielski \\ Universidade Federal do Paraná (UFPR)
}

Fecha de Recepción: 9 Febrero 2018

Fecha de Admisión: 10 Abril 2018

\begin{abstract}
RESUMO
Compreender as crenças sobre as práticas parentais, pode fornecer elementos aos profissionais na atuação junto à criança nos anos iniciais da infância e à sua família, elaborando estratégias efetivas de Intervenção Precoce. 0 objetivo foi investigar as crenças sobre as práticas parentais em famílias de crianças com atrasos no desenvolvimento. Participaram 36 famílias de 40 crianças que frequentam a Educação Infantil e apresentam diferentes atrasos em seu desenvolvimento, da cidade de Curitiba, Brasil. Foi realizada uma visita domiciliar para cada família, em que os pais responderam a dois questionários. Entre os resultados, verificou-se que quanto maior nível de importância às crenças sobre práticas de Apresentação da criança em público, menor é o grau de escolaridade parental. Conclui-se que as crenças sobre as práticas parentais se diferenciam quando relacionadas a outros fatores, em especial com a escolaridade parental e presença de práticas religiosas, que mostraram elevada importância para esta amostra.
\end{abstract}

Palavras-chave: relações familiares; desenvolvimento infantil; intervenção precoce

\section{ABSTRACT}

Beliefs on parental practices in families of children with developments in development.

Understanding beliefs about parental practices can provide practitioners of early child care and their families elements for the design of effective early intervention strategies. The present study aimed to investigate the beliefs about parental practices in families of children with developmental delays. Thirty-six families of 40 children attending the Municipal Early Childhood Centers, who presented different delays in their development, in the city of Curitiba, Brazil, participated in this study the home visit was conducted for each family, in which the parents answered the two questionnaires. Among the results, it was verified that the higher the level of importance to the beliefs about prac- 
tices of presentation of the baby or child in public, the lower the level of parental schooling. From these results, it is concluded that beliefs about parental practices differ when related to other factors, especially with parent level of education, and the presence of religious practices, which showed high importance for this sample.

Keywords: cognitions; family relations; childhood development; early intervention

0 tema das crenças sobre práticas parentais, e seu papel no desenvolvimento infantil, tem sido amplamente pesquisado (Harkness \& Super, 1996; Kobarg \& Vieira, 2008; Silva \& Magalhães, 2011; Vieira et al., 2010). Estudos como os de Harkness e Super (1996) e Kobarg e Vieira (2008) reforçam que as crenças atuam como base para o comportamento das pessoas, e estas crenças se encontram mais subentendidas do que claramente expressas nos comportamentos parentais.

Buscar compreender as crenças parentais facilitará uma representação das cognições e do próprio desenvolvimento do cuidador, podendo revelar o trajeto para contribuir na compreensão das atividades realizadas pelos pais. Elas são capazes de fornecer interpretações sobre o contexto em que a criança está inserida, e assim exercer influência no andamento das transmissões culturais (Goodnow, 1992; Harkness \& Super, 1996). Deste modo, o termo "crenças sobre práticas parentais" é definido como as ideias, princípios ou interpretações realizadas por parte dos pais em relação à relevância de determinadas práticas de cuidado com seus filhos (Silva, 2008).

Em diversas pesquisas, o fator nível de escolaridade tem tido destaque como um dos elementos que justificam possíveis diferenças entre as crenças parentais e 0 desenvolvimento da criança (Keller et al., 2006; Kobarg \& Vieira, 2008; Ribas, Seidl de Moura \& Bornstein, 2003, 2007; Silva \& Magalhães, 2011; Suizzo, 2002). No estudo de Silva e Magalhães (2011) verificou correlação significativa entre a escolaridade materna e as crenças parentais voltadas à estimulação, ou seja, quanto maior a escolaridade na mãe, mais ela tende a valorizar práticas voltadas à estimulação em várias áreas do desenvolvimento de seu filho (como a motora, cognitiva, linguagem, entre outras).

As crenças sobre práticas parentais também têm sido exploradas em relação às crianças com diferentes formas de atraso no desenvolvimento (Amparo-Sobrinho, 2010; Minetto, 2010; Minetto \& Löhr, 2016; Portes, 2013). 0 termo atraso no desenvolvimento pode ser compreendido como uma categoria e/ou condição temporária, provisória ou decisiva em relação a um determinado prejuízo no desenvolvimento da criança, seja em uma área específica do desenvolvimento, ou até para se referir a um diagnóstico (Dornelas, Duarte \& Magalhães, 2015; Marín, Esteban \& Bañón, 2015).

A Intervenção Precoce (IP) visa garantir maior qualidade ao desenvolvimento da criança, que neste processo é compreendida em sua totalidade, na busca por trabalhar com a família no potencial deste desenvolvimento. Este programa centrado na família favorece aos profissionais da IP em perceber deficiências presentes no contexto destas famílias, a saber: qualidade do ambiente e da relação familiar, práticas de cuidado, integração da criança e sua família aos recursos sociais, entre outros (Franco, 2015).

Bronfenbrenner (2011) descreve que, entre as pessoas e os contextos físicos e sociais, há uma inter-relação de reciprocidade. 0 contexto familiar, no qual há uma influência mútua na relação entre seus integrantes (pai, mãe e filhos), acabam por receber influência de outras pessoas que podem ou não estar neste contexto de interação. Uma investigação em torno da relação parental (díade primária) pode fornecer maior clareza das alterações no desenvolvimento dos filhos, assim como compreender o desenvolvimento dos cuidadores e do ambiente (Kobarg \& Vieira, 2008; Yunes \& Juliano, 2010).

Buscar compreender o desenvolvimento de crianças que frequentam a rede de educação infan- 
til possibilita ações mais efetivas de IP na prática de profissionais, pesquisadores e no trabalho realizado com as famílias. Deste modo, o presente estudo teve por objetivo investigar as crenças sobre as práticas parentais em famílias de crianças com atrasos no desenvolvimento.

\section{MÉTODO}

\section{Participantes}

Participaram desta pesquisa 36 famílias, das quais foram selecionados os pais e mães de 40 crianças ( $77,5 \%$ eram do gênero masculino e outros $22,5 \%$ do gênero feminino), tendo essas crianças idades entre 15,5 meses (aproximadamente um ano e três meses) e 68,4 meses (aproximadamente 5 anos e 10 meses), perfazendo uma média de idade em 48,33 meses (aproximadamente 4 anos) e um desvio-padrão de 12,78 meses (aproximadamente um ano e oito meses). As crianças frequentavam os Centros Municipais de Educação Infantil (CMEl's) na cidade de Curitiba, região Sul do Brasil.

A maioria dos pais participantes tinha ensino fundamental incompleto $(40 \%)$ ou ensino médio completo (25\%), $15 \%$ dos participantes apresentaram ensino superior completo e $10 \%$ ensino superior incompleto, outros 7,5\% ensino médio incompleto e 2,5\% ensino fundamental completo. Entre as mães participantes, a maioria possuía ensino médio completo (30\%) ou ensino fundamental incompleto (27,5\%), 20\% das participantes tinham ensino superior completo e 7,5\%, igualmente, ensino fundamental completo e ensino médio incompleto, outros $5 \%$ ensino superior incompleto e $2,5 \%$ eram analfabetas.

Verificou-se um número médio de 4,3 pessoas por família, com um desvio-padrão de 1,655. Quanto ao status civil desses participantes, $40 \%$ possuíam união estável, $30 \%$ viviam juntos, $20 \%$ eram separados/divorciados e outros $10 \%$ estavam casados. Em relação à religião predominante entre as famílias, 42,5\% declararam pertencer à religião Evangélica, 35\% à Católica, 10\% não tinham religião, 7,5\% não eram praticantes, e mesma porcentagem de 2,5\% para os que seguiam a religião Espírita e Outras.

\section{Instrumentos}

Questionário Sociodemográfico: identificação e composição das famílias participantes, com breve caracterização de dados sociodemográficos e do ambiente familiar.

Questionário de Crenças e Ideias sobre Bebês e Crianças Pequenas: adaptado para a população brasileira no estudo de Vieira et al. (2010), a partir do original desenvolvido por Suizzo (2002), denominado Croyances es idées sur lês nourissons et petits enfants (CINPE) ou Beliefs and Ideas about Infants and Young Children, que busca identificar crenças sobre as práticas parentais de cuidados em bebês e crianças pequenas. 0 instrumento contém 50 itens, dos quais 25 estão relacionados a bebês menores de 1 ano de idade e os outros 25 a bebês entre 1 a 3 anos de idade. Os pais são convidados a responder cada um dos itens de acordo com o que "pensa" e não de acordo com 0 que "faz" e/ou se já "fez" com seu filho (a). E assim, o cuidador assinalar, classificando o nível de importância atribuído à prática, usando uma escala de Likert de 6 pontos $(0=$ discordo, $1=$ sem importância, 2 = de pouca importância, 3 = de média importância, 4 = muito importante, e 5 = extremamente importante). Desta forma é possível verificar estas crenças sobre as práticas parentais em quatro dimensões referentes a diferentes domínios do cuidado parental: 1) Estimulação (com a exposição da criança para diferentes formas de estímulos que alcancem áreas do desenvolvimento cognitivo e/ou motor); 2) Apresentação apropriada do bebê/criança (assegurar a apresentação adequada da criança em diferentes contextos e/ou situações); 3) Responsividade (busca em compreender e vincular-se à criança); e 4) Disciplina (oferecer diferentes formas de controle rígido à 
criança). A correção foi realizada com a soma de todos os itens correspondentes à cada dimensão avaliada, gerando um escore bruto e percentual para cada dimensão. Importante salientar que a presente pesquisa tem em sua amostra crianças acima de 5 anos de idade. Os participantes com crianças acima dos 3 anos de idade já vivenciaram a fase de desenvolvimento proposta pelo instrumento e, ao responderem ao questionário, foi possível mencionar acontecimentos relacionados à idade inferior a 3 anos da criança.

\section{Procedimentos}

0 projeto foi submetido e aprovado pelo Comitê de Ética, com parecer de ํㅜㄴ 1.573.473. A indicação das famílias foi realizada pela Coordenadoria de Atendimento as Necessidades Especiais (CANE) e por CMEl's visitados pelos pesquisadores. Com os dados das famílias (de crianças que apresentavam algum atraso no desenvolvimento), os pesquisadores agendaram uma data e horário para visitar a casa das famílias que aceitaram o convite em participar da pesquisa. Durante a visita, foi feita a leitura e recolhida assinatura do Termo de Consentimento Livre e Esclarecido (TCLE). Após a assinatura, iniciou-se a coleta de dados por meio de entrevista.

\section{Análise de Dados}

Utilizou-se estatística descritiva, em que os testes estatísticos foram calculados com o software IBM SPSS Statistics na versão 21.0. A análise dos itens do questionário de crenças sobre práticas parentais foi realizada de acordo com os quatro fatores indicados por Suizzo (2002): 1) Estimulação (itens de 1 a $16-\alpha=0,633$ ); 2) Apresentação (itens de 17 a $24-\alpha=0,582$ ); 3) Responsividade (itens de 25 a 32, com a inclusão dos itens 37, 38 e $40-\alpha=0,659$ ); e 4) Disciplina (itens $33,36,37,39$, e $41-\alpha=0,541$ ). Os itens restantes 42 até 50 não foram incluídos respeitando as análises fatoriais indicadas por Vieira et al. (2010).

\section{RESULTADOS}

Com a realização do cálculo da Média $(m)$ e do Desvio-Padrão $(d p)$ das dimensões relacionadas às crenças sobre práticas parentais, as famílias desta amostra atribuíram elevado valor de importância à dimensão de Estimulação ( $m=70,87 ; d p=5,38)$, seguida da dimensão de Disciplina $(m=$ $42,70 ; d p=6,48)$. Contudo, apareceram com razoável importância as dimensões de Apresentação $(m=35,70 ; d p=3,51)$ e Responsividade $(m=35,10 ; d p=5,30)$. Esses dados podem ser visualizados na Tabela 1.

Tabela 1: Escores (em termos de Média e Desvio-Padrão) alcançados pelas famílias participantes em Relação às Dimensões do Questionário de Crenças e Ideias sobre Bebês e Crianças Pequenas

\begin{tabular}{lcc}
\hline \multicolumn{1}{c}{ Dimensões } & $\boldsymbol{m}$ & $\boldsymbol{d p}$ \\
\hline Estimulação & 70,87 & 5,38 \\
Apresentação & 35,70 & 3,51 \\
Responsividade & 35,10 & 5,30 \\
Disciplina & 42,70 & 6,48 \\
\hline
\end{tabular}

Nota. $m=$ média. $d p=$ desvio padrão.

Na análise de correlação $r$ de Pearson entre as dimensões do questionário, as dimensões de Estimulação e Responsividade foram correlacionadas e constatou-se uma correlação significativa, positiva e fraca $(r=0,315 ; p<0,048)$. Ou seja, quanto maiores as crenças sobre práticas relacionadas à Estimulação, maiores são as crenças sobre práticas ligadas à Responsividade das famílias para com a criança. 
Foi encontrada correlação positiva, significativa e moderada entre as dimensões de Apresentação e Responsividade ( $r=0,533 ; p<0,001)$, pode-se interpretar que, quanto maior for a preocupação por parte da família com a Apresentação adequada da criança em público, maiores serão as respostas parentais para os sinais da criança (Responsividade). A mesma correlação ocorreu entre as dimensões de Estimulação e Disciplina, $(r=0,419 ; p<0,007)$.

Para as dimensões de Responsividade e Disciplina, houve uma correlação significativa, positiva e de magnitude forte $(r=0,730 ; p<0,001)$, e de forma análoga ocorreu com a correlação das dimensões de Apresentação e Disciplina $(r=0,677 ; p<0,000)$. A Tabela 2 apresenta os dados acima citados.

Tabela 2: Correlações entre as Dimensões do Questionário de Crenças e Ideias sobre Bebês e Crianças Pequenas

\begin{tabular}{lccr}
\hline \multicolumn{1}{c}{ Dimensões } & Estimulação & Apresentação & Responsividade \\
\hline Apresentação & 0,198 & & \\
Responsividade & $0,315^{*}$ & $0,574^{* *}$ & \\
Disciplina & $0,419^{* *}$ & $0,677^{* *}$ & $0,730^{* *}$ \\
\hline
\end{tabular}

Nota. ${ }^{*} \mathrm{p}<0,05 ;{ }^{* *} \mathrm{p}<0,01$.

Realizada a análise de correlação $r$ de Pearson entre as dimensões do questionário com os aspectos sociodemográficos (escolaridade dos pais e número de pessoas que compõem a família), constatou-se entre a dimensão de Apresentação e o aspecto de Escolaridade Materna uma correlação significativa, negativa e fraca $(r=-0,312 ; p>0,05)$. Isto é, quanto maior 0 grau de importância das crenças sobre práticas de Apresentação apropriadas ao bebê ou criança em público, menor é 0 grau de escolaridade das mães. 0 mesmo tipo de correlação ocorreu entre esta dimensão e a da Escolaridade Paterna $(r=-0,345 ; p>0,03)$.

Foi verificada correlação significativa, negativa e moderada entre as variáveis de escolaridade materna $(r=-0,418 ; p<0,007)$ e escolaridade paterna $(r=-0,458 ; p<0,003)$ com relação ao número de pessoas que compõem a família. Esses dados podem ser visualizados na Tabela 3.

Tabela 3: Correlações entre os Dados Sociodemográficos e as Dimensões do Questionário de Crenças e Ideias sobre Bebês e Crianças Pequenas

\begin{tabular}{lrr}
\hline \multicolumn{1}{c}{ Dados Sociodemográficos / Dimensões } & EM & \multicolumn{1}{l}{ EP } \\
\hline Número de pessoas que compõe a família & $-0,418^{* *}$ & $-0,458^{* *}$ \\
Apresentação & $-0,312^{* *}$ & $-0,345^{* *}$ \\
\hline
\end{tabular}

Nota. ${ }^{\text {a }}$ escolaridade materna (EM); ${ }^{\mathrm{b}}$ escolaridade paterna (EP); $1=$ analfabeta (não alfabetizada); $2=$ fundamental incompleto (até a $4^{\circ}$ série); $3=$ fundamental completo (até a $8^{\circ}$ série); $4=$ ensino médio incompleto; $5=$ ensino médio completo; $6=$ superior incompleto; $7=$ superior completo. ${ }^{*} \mathrm{p}<0,05 ; * * \mathrm{p}$ $<0,01$.

Ao realizar o teste de diferença de médias $t$ de Student para comparar as dimensões do questionário com o status civil (divididos em dois grupos: casados e separados/divorciados) das famílias, a dimensão Responsividade quando comparada com o grupo de pais casados (legalmente casados, união estável e vivem juntos) e o grupo de pais separados/divorciados, encontrou-se diferença estatisticamente significativa entre estes dois grupos $(t=2,739 ; p=0,009)$. Ou seja, observou-se que os pais casados mantêm, de modo geral, maior Responsividade com a criança do que os pais separados/divorciados. A Figura 1 apresenta um diagrama de caixas (boxplot) ilustrando os dados citados acima. 
Figura 1

Diagrama de caixas comparando casados e separados/divorciados em relação à dimensão Responsividade. Elaborado pelos autores.

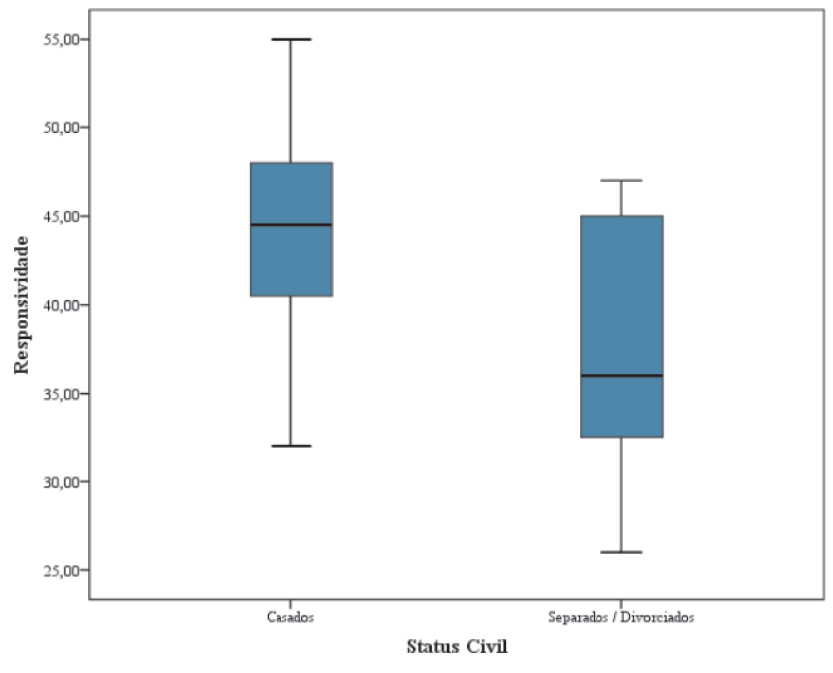

0 mesmo teste de diferença de médias $t$ de Student foi realizado para comparar as dimensões avaliadas com 0 aspecto de religião (a amostra foi dividida em dois grupos: com prática religiosa e sem prática religiosa) na família. A dimensão Apresentação, quando comparada com o grupo de pais com prática religiosa (Evangélicos, Católicos, Espíritas e outros) e o grupo de pais sem prática religiosa (não tinham religião e não eram praticantes), constatou-se diferença estatisticamente significativa entre estes dois grupos $(t=2,658 ; p=0,011)$. Houve diferença estatisticamente significativa quando estes dois grupos foram comparados com a dimensão Disciplina $(t=3,152 ; p=$ $0,004)$. Encontrou-se também diferença estatisticamente significativa quando estes dois grupos de pais foram comparados com a dimensão Responsividade $(t=2,064 ; p=0,046)$.

\section{DISCUSSÃO}

Por meio da análise de dados, foram encontradas diferenças significativas nas crenças sobre práticas parentais das famílias desta amostra. E entre as dimensões avaliadas, constatou-se maior grau de importância para a dimensão de Estimulação da criança. Dado também constatado por Suizzo (2002) com uma amostra de pais e mães parisienses de crianças de desenvolvimento típico, em que estas famílias valorizavam mais (em média) as crenças sobre práticas parentais voltadas à Estimulação de seus filhos. Portes (2013), ao investigar uma amostra de famílias de crianças com SD, em relação à importância atribuída às práticas de Estimulação, constatou que mães investem maior importância às práticas ligadas a esta dimensão do que de fato realizam com seus filhos.

A preocupação sob a Estimulação por parte dos entrevistados se dá porque a maior parte das crianças da amostra já possui algum diagnóstico, enquanto outra parte revela suspeitas e presença de diferentes atrasos no desenvolvimento. Tal dado pode levar a família em um processo de IP. Franco (2015) salienta que a IP acontece somente quando a equipe profissional ajuda a família a reconhecer a criança em toda sua totalidade. E desta forma, pode auxiliar a família a não apenas esti- 
mular os problemas evidentes na criança, mas também assegurar um ambiente familiar com condições facilitadoras para o desenvolvimento da criança.

Um dos aspectos que apresentaram resultado significativo foi a escolaridade dos participantes adultos. Esta informação vai ao encontro de outras pesquisas relacionadas às crenças sobre práticas parentais, indicando que o nível de ensino interfere significativamente na interação entre família e criança, assim como no desenvolvimento infantil (Kobarg \& Vieira, 2008; Portes, 2013; Ribas et al., 2003; Ribas, Seidl de Moura \& Bornstein, 2007; Seidl de Moura et al., 2004; Seidl de Moura et al., 2008; Seidl de Moura, Ribas, Piccinini et al., 2004; Suizzo, 2002; Silva \& Magalhães, 2011; Sobrinho, 2010).

Os resultados obtidos das correlações efetuadas entre as variáveis sociodemográficas, em especial o número de pessoas que residem no ambiente familiar com a variável escolaridade parental, mostrou correlação estatisticamente significativa e negativa, ou seja, quanto menor for o número de pessoas na residência, maior será a escolaridade dos pais. Diante disto, a literatura tem mostrado, de forma análoga, que baixa escolaridade e elevado número de pessoas na residência são fatores de risco ao desenvolvimento da criança. Os fatores de risco incluem características da criança, da família e do ambiente, propiciando a redução nas habilidades parentais e, consequentemente, diminuindo oportunidades relacionais ao bom desenvolvimento da criança (Silva, Nunes, Betti \& Rios, 2008). Sendo assim, a teoria Bioecológica (Bronfenbrenner, 2011) descreve que baixa escolaridade e maior número de pessoas na residência são variáveis distais no contexto imediato da criança, e interferem diretamente nas inter-relações que ocorrem no microssistema familiar e afetam o desenvolvimento dos filhos.

Os participantes da amostra foram divididos em dois grupos em relação ao status civil dos pais, os resultados apontaram que pais casados mantêm, de modo geral, maior Responsividade com a criança do que os pais separados/divorciados. A dimensão de Responsividade está ligada diretamente com elementos afetivos e tempo dispensado na relação com a criança, como intimidade, proximidade e afeto positivo (Ribas, Seidl de Moura \& Ribas, 2003).

Os pais ao se separar, o cuidador que fica responsável pelos cuidados da criança (as mães são responsáveis em grande maioria), suas práticas parentais tendem a ser menos efetivas e dificuldades para responder aos sinais da criança com diminuição das habilidades de resolução de problemas. Assim, os filhos estão mais vulneráveis, principalmente os mais novos, que dependem mais dos cuidados parentais, visto que o processo de separação conjugal é complexo, dificultando a recolocação do papel parental quanto aos cuidados com a criança (Amato, 2005; Martinez \& Forgatch, 2002; Strohshein, 2005).

Outra variável que apresentou destaque entre os resultados, quando correlacionada com as dimensões de Apresentação, Responsividade e Disciplina, foi a prática religiosa. Os participantes que relataram pertencer ao grupo de pais com prática religiosa apresentaram, em relação ao grupo de pais sem prática religiosa, maior preocupação com a apresentação da criança em público, maior envolvimento com a criança e práticas voltadas à disciplina da criança. Estes dados podem ser discutidos com os estudos de Bruscagin (2013), que reconhecem, entre as pessoas que têm alguma religião, uma maior compreensão dos acontecimentos da vida (de um modo geral), estruturada de acordo com suas crenças. Henning-Geronasso e Moré (2009) reforçam que religiosidade tem impacto positivo em pessoas que experimentam grande estresse, como famílias com filhos com atrasos no desenvolvimento.

Concluindo, é importante reconhecer que grande parte dos pais e mães tem crenças parentais pautadas em uma educação tradicional (práticas de bater e ameaçar a criança como métodos de educar), da mesma forma como as recebeu de seus progenitores, o que acaba por prejudicar a utilização de práticas parentais adequadas. 
As crenças parentais podem propiciar práticas adequadas de cuidado com a criança. Mas, para que isto ocorra, é fundamental que profissionais busquem reconhecer não só as características da criança, e sim todos os aspectos que envolvem o complexo sistema familiar. Deste modo, procurar implantar estratégias de intervenção juntamente às famílias, com o propósito de viabilizar aos pais reflexão sobre os modos de cuidados e de educação dispensados às crianças, e tornem-se capazes de assumir estratégias educativas que beneficiem o desenvolvimento de seus filhos.

Destaca-se que 0 conhecimento, advindo das crenças sobre práticas parentais, apresenta grande potencial para subsidiar estratégias profissionais efetivas, com 0 trabalho voltado à promoção do desenvolvimento infantil. 0 conhecimento sobre as crenças que pais e mães têm sobre desenvolvimento infantil e as crenças que estes possuem sobre práticas de cuidado na criação e a educação de seus filhos podem favorecer o exercício profissional dentro de um processo de IP.

0 sistema de crenças parentais está relacionado diretamente com os sistemas culturais estabelecidos no contexto com que a família mantém interação, e que refletem as práticas parentais. Nesta pesquisa foi possível constatar que características como a escolaridade da mãe e do pai, status civil, número de pessoas que residem no ambiente familiar e religiosidade interferem significativamente nas crenças sobre práticas parentais. Para futuros estudos, sugere-se utilizar amostras de diferentes contextos, que apresentem perfil sociocultural distinto, a fim de maiores comparações.

\section{REFERÊNCIAS}

Amato, P. R. (2005). The impact of family formation change on the cognitive, social, and emotional well-being of the next generation. The Future of Children, 15 (2), 75-96. doi: $10.1353 /$ foc. 2005.0012

Amparo-Sobrinho, L. (2010). O cuidado a crianças com síndrome de Down sob a perspectiva evoIucionista. Dissertação de Mestrado, Universidade Federal da Bahia, Salvador-BA.

Bronfenbrenner, U. (2011). Bioecologia do desenvolvimento humano: Tornando os seres humanos mais humanos. (A. Carvalho-Barreto, Trad.). Porto Alegre: Artmed. (Trabalho original publicado em 2005).

Bruscagin, C. (2013). Família e religião. Em: C. M. O. Cerveny (Org.), Família e... Comunicação, divórcio, mudança, resiliência, deficiência, lei, bioética, doença, religião e drogadição (pp. 163186). São Paulo: Casa do Psicólogo.

Dornelas, L. F., Duarte, N. M. C., \& Magalhães, L. C. (2015). Atraso do desenvolvimento neuropsicomotor: Mapa conceitual, definições, usos e limitações do termo. Revista Paulista de Pediatria, 33, 88-103. doi: 10.1016/j.rpped.2014.04.009

Franco, V. (2015). Introdução á intervenção precoce no desenvolvimento da criança: Com a família, com a comunidade, em equipe. Évora: Edições Aloendro.

Goodnow, J. (1992). Parents ideas, childrens ideas: Correspondence and divergence. Em I. E. Sigel, A. V. McGillicuddy-DeLisi \& J. J. Goodnow (Orgs.), Parental belief systems: The psychological consequences for children (pp. 293-317). Hillsdale: Erlbaum.

Harkness, S., \& Super, C. M. (1996). Parents' cultural belief systems: Their origins, expressions, and consequences. Nova York: Guilford.

Henning-Geronasso, M. C., \& Moré, C. L. O. 0. (2009). Influência da religiosidade/ espiritualidade no contexto psicoterapêutico. Psicologia: Ciência e Profissão, 35 (3), 711-725. doi: 10.1590/1982-3703000942014

Keller, H., Lamm, B., Abels, M., Yovsi, R., Borke, J., Jensen, H., et al. (2006). Cultural models, socialization goals, and parenting ethnotheories: A multicultural analysis. Journal of Cross-Cultural Psychology, 37 (2), 155-172. doi: 10.1177/0022022105284494 
Kobarg, A. P., \& Vieira, M. L (2008). Crenças e práticas de mães sobre desenvolvimento infantil nos contextos rural e urbano. Psicologia Reflexão e Crítica, 21 (3), 401-408. doi: 10.1590/S010279722008000300008

Marín, F. A., Esteban, Y. A., \& Bañón, D. R. (2015). Herramientas de cribado para la detección de retrasos o trastornos en el desarrollo: Una revisión sistemática de la literatura. Revista Española de Discapacidad, 3 (2), 7-26. doi: 10.5569/2340-5104.03.02.01

Martinez, C. R. J., \& Forgath, M. S. (2002). Adjusting to change: Linking family structure transitions with parenting boys adjustment. Journal of Family Psychology, 16 (2), 107-117. doi: 10.1037/0893-3200.16.2.107

Minetto, M. F. J. (2010). Práticas educativas parentais, crenças parentais, estresse parental e funcionamento familiar de pais de crianças com desenvolvimento típico e atípico. Tese de Doutorado, Universidade Federal de Santa Catarina, Florianópolis-SC.

Minetto, M. F., \& Löhr, S. S. (2016). Crenças e práticas educativas de mães de crianças com desenvolvimento atípico. Educar em Revista, 59, 49-64. doi: 10.1590/0104-4060.44791

Portes, J. R. M. (2013). Crenças sobre práticas de cuidado e metas de socialização de pais e mães com filhos com síndrome de Down. Dissertação de Mestrado, Universidade Federal de Santa Catarina, Florianópolis-SC.

Ribas, A. F. H., Seidl de Moura, M. L., \& Ribas., R. C. (2003). Responsividade materna: Levantamento bibliográfico e discussão conceitual. Psicologia: Reflexão e Crítica, 16, 137-145. doi: 10.1590/S0102-79722003000100014

Ribas, R. C., Seidl de Moura, M. L., \& Bornstein, M. H. (2003). Socioeconomic status in Brazilian psychological research. Part 2: SES and parenting knowledge. Estudos de Psicologia, 8, 385392. doi: 10.1590/S1413-294X2003000300005

Ribas, R. C., Seidl de Moura, M. L., \& Bornstein, M. H. (2007). Cognições maternas acerca da maternidade e do desenvolvimento humano: Uma contribuição ao estudo da psicologia parental. Revista Brasileira de Crescimento e Desenvolvimento Humano, 17, 104-113. doi: 10.7322/jhgd.19819

Seidl de Moura, M. L., Ribas, A. F. P., Seabra, K. C., Pessôa, L. F., Ribas, R. C., \& Nogueira, S. E. (2004). Interações iniciais mãe-bebê. Psicologia: Reflexão e Crítica, 17 (3), 295-302. doi: 10.1590/S0102-79722004000300002

Seidl de Moura, M. L., Ribas, A. F. P., Seabra, K. C., Pessôa, L. F., Nogueira, S. E., Mendes, D. M. L. F., et al. (2008). Interações mãe-bebê de um e cinco meses: Aspectos afetivos, complexidade e sistemas parentais predominantes. Psicologia: Reflexão e Crítica, 21, 66-73. doi: 10.1590/S0102-79722008000100009

Seidl de Moura, M. L., Ribas, R. C., Piccinini, C. A., Bastos, A. C. S., Magalhães, C. M. C., Vieira, M. L., M. L., et al. (2004). Conhecimento sobre desenvolvimento infantil em mães primíparas de diferentes centros urbanos do Brasil. Estudos de Psicologia, 9 (3), 421-429. doi:10.1590/S1413-294X2004000300004

Silva, N. C. B., Nunes, C. C., Betti, M. C. M., \& Rios, K. S. A. (2008). Variáveis da família e seu impacto sobre 0 desenvolvimento infantil. Temas em Psicologia, 16 (2), 215-229. "(DOI INEXISTENTE)"

Silva, R. A. (2008). Cognições parentais: Crenças, metas e estratégias de socialização de mães primíparas. Dissertação de mestrado, Universidade Federal do Pará, Belém-PA.

Silva, R. A., \& Magalhães, C. M. C. (2011). Crenças sobre práticas: Um estudo sobre mães primíparas de contexto urbano e não-urbano. Revista Brasileira Crescimento Desenvolvimento Humano, 21, 39-50. "(DOI INEXISTENTE)" 
Sobrinho, L. A. (2010). 0 cuidado parental de crianças com síndrome de Down sob a perspectiva evolucionista. Dissertação de Mestrado, Universidade Federal da Bahia, Salvador-BA.

Suizzo, M. A. (2002). French parent's cultural models and childrearing beliefs. International Journal of Behavioral Development, 26 (4), 297-307. doi: 10.1080/01650250143000175

Vieira, M. L., Seidl de Moura, M. L., Lordelo, E., Piccinini, C., Martins, G. D. F., Macarini, S. M., et al. (2010). Mother's beliefs about childrearing practices in seven Brazilian cities. Journal of Cross-Cultural Psychology, 41 (2), 195-211. doi: 10.1177/0022022109354642

Yunes, A. M., \& Juliano, M. C. (2010). A biecologia do desenvolvimento humano e suas interfaces com educação ambiental. Cadernos de Educação, 37, 347-379. "(DOI INEXISTENTE)” 